\title{
Cognitive Predictors of Delirium on Long-Term Follow-Up after TIA and Stroke: Population-Based Cohort Study
}

\author{
Sarah T. Pendlebury a,b,c Ross J. Thomson ${ }^{d}$ Sarah J.V. Welch ${ }^{a}$ \\ Peter M. Rothwell ${ }^{\mathrm{a}}$ Oxford Vascular Study \\ aWolfson Centre for Prevention of Stroke and Dementia, Wolfson Building, Nuffield Department of Clinical \\ Neurosciences, John Radcliffe Hospital, Oxford, UK; ${ }^{b}$ NIHR Oxford Biomedical Research Centre, Oxford University \\ Hospitals NHS Foundation Trust, Oxford, UK; ' $D$ Departments of General (Internal) Medicine and Geratology, Oxford \\ University Hospitals NHS Foundation Trust, Oxford, UK; ${ }^{\mathrm{d} D e p a r t m e n t ~ o f ~ C a r d i o l o g y, ~ R o y a l ~ F r e e ~ H o s p i t a l, ~ L o n d o n, ~ U K ~}$
}

\section{Keywords}

Delirium · TIA and stroke $\cdot$ Cognitive impairment $\cdot$ Longterm follow-up

\begin{abstract}
Introduction: TIA and stroke cause cognitive impairment with a typical "vascular" pattern, including prominent frontal/executive deficits. Cognitive impairment is associated with increased delirium risk and the few available data suggest that executive dysfunction is important. We therefore determined the predictive value of both severity and pattern of cognitive deficits for delirium on long-term follow-up after TIA/stroke. Methods: Surviving TIA/stroke participants on October 1, 2013, in the Oxford Vascular Study (OXVASC) were assessed prospectively for delirium during all hospitalizations over the subsequent 6 months. Associations between OXVASC pre-admission mini-mental state examination (MMSE) and Montreal Cognitive Assessment (MoCA) scores, and delirium during hospitalizations on follow-up were determined using logistic regression adjusted for covariates, including demographic factors, history of depres-
\end{abstract}

sion, baseline stroke severity, and admission illness severity. Results: Among 1,565 TIA/stroke survivors, 158 patients (mean/SD age $=79.2 / 11.5$ years) had $\geq 1$ admission and 59 (37\%) had $\geq 1$ delirium episode. Mean/SD time between baseline TIA/stroke and admission was 4.7/3.6 years and between most recent OXVASC cognitive testing and admission was 1.7/1.8 years. MMSE and MoCA scores were associated with delirium: odds ratio $(\mathrm{OR})=1.16(95 \% \mathrm{Cl} 1.07-1.27, p<$ 0.0001 per point decrease in MMSE) and OR $=1.20$ (1.11$1.30, p<0.0001 \mathrm{MoCA}$ ) and associations were robust to adjustment for all covariates, including stroke severity: $O R=$ $1.11(1.01-1.22, p=0.03, \mathrm{MMSE})$ and $\mathrm{OR}=1.15$ (1.05-1.25, $p=0.003, \mathrm{MoCA}$ ). All 10 subtests on the MoCA and 4/11 on the MMSE were significantly associated with delirium with highest predictive value for frontal/executive and recall domains. Conclusions: Cognitive impairment of increasing severity after TIA/stroke predisposed to delirium particularly deficits in frontal/executive domains and recall. Long-term risk of delirium should be considered as part of the overall cerebrovascular disease burden.

(C) 2021 The Author(s)

Published by S. Karger AG, Basel karger@karger.com www.karger.com/ced

Karger $\stackrel{\text { '⿳亠丷厂 }}{ }$

BOPEN ACCESS
(C) 2021 The Author(s)

Published by S. Karger AG, Basel

This article is licensed under the Creative Commons Attribution 4.0 International License (CC BY) (http://www.karger.com/Services/ OpenAccessLicense). Usage, derivative works and distribution are permitted provided that proper credit is given to the author and the original publisher.
Correspondence to:

Sarah T. Pendlebury, sarah.pendlebury@ clneuro.ox.ac.uk 


\section{Introduction}

Pre-existing cognitive impairment predisposes to delirium $[1,2]$. Stroke causes cognitive decline and dementia in proportion to its severity which may explain why a history of stroke is associated with increased delirium risk in general hospital cohorts [1-3]. The pattern of neurocognitive deficits may also be important: executive dysfunction has been postulated to increase delirium possibly through altered brain networks and reduced ability to integrate sensory inputs $[4,5]$. Although stroke-associated cognitive impairment is heterogeneous depending on stroke location and the underlying neuropathology, a characteristic "vascular pattern" of deficits is seen, including relatively prominent frontal/executive dysfunction [6-8]. This cognitive impairment profile might therefore also increase the likelihood of delirium after stroke.

Delirium in the acute phase of stroke has shown to be associated with pre-existing cognitive impairment, as well as with stroke severity and infection [9], but there are few studies of delirium in the longer term after cerebrovascular events. In a population-based study, we determined associations between the severity and pattern of cognitive domain deficits occurring after TIA and stroke, and future delirium occurring during hospitalization on long-term follow-up.

\section{Methods}

\section{Oxford Vascular Study}

The patients in the current study were participants with TIA or stroke previously recruited since 1 April 2002 in the Oxford Vascular Study (OXVASC), an ongoing longitudinal populationbased cohort study within a population of $>92,700$ covered by 9 primary care practices ( 100 primary care practitioners) in Oxfordshire, UK [10]. The study was approved by the local research Ethics Committee. Informed written consent (or assent from relatives) was obtained for study interview and face-to-face and telephone follow-up as well as indirect follow-up using medical records.

TIA and stroke were defined clinically by WHO criteria and index cerebrovascular event severity was measured using the National Institutes of Health Stroke Scale (NIHSS) [10]. Patient data were collected by interview using a standardized form and from general practitioner records. Follow-up interviews were done by clinical fellows and trained research nurses at 1 and 6 months and 1,5 , and 10 years either in the out-patient clinic or by home visit. Cognitive testing was conducted at baseline and all follow-ups (Fig. 1) using one or more of mini-mental state examination (MMSE) [11] and Montreal Cognitive Assessment (MoCA) [12] validated for use in TIA/stroke [8].
Prospective Ascertainment of Delirium

The Oxford University Hospitals NHS Foundation Trust (OUHFT) provides acute services for the population of approximately 660,000 in Oxfordshire, including all patients in the OXVASC study primary care practises. OXVASC patients requiring acute hospital care for any reason are admitted either to OUHFT or to Abingdon Emergency Medical Unit (EMU). To identify OXVASC participants admitted to hospital over the study period, we conducted daily searches of electronic (OUHFT) and paper records (EMU) for all new admissions excluding day case procedures, and cross-checked these against the register of OXVASC participants.

All TIA and stroke participants in OXVASC surviving on October 1,2013 , were included in the current study. Subsequent hospital admissions for any reason were prospectively identified from October 2013-April 2014 at the OUHFT and the Abingdon EMU. Patients were assessed as soon as possible after admission by members of the OXVASC study team. Delirium was ascertained using gold standard clinical diagnosis rather than retrospective hospital administrative diagnostic (ICD-10)-coded data since the latter are insensitive [13].

Delirium diagnosis was made according to the DSM-IV [14] criteria by STP after discussion with the OXVASC study team supplemented by review of the medical notes, including the OUHFT cognitive screen as described previously $[2,13]$ (see online suppl. methods; for all online suppl. material, see www.karger.com/ doi/10.1159/000519900). The OUHFT cognitive screen is mandated on all patients admitted to the OUHFT or EMU who are aged $\geq 70$ years or $<70$ years with confusion/altered behaviour and includes the 10-point abbreviated mental test score (AMTS) [15] together with the Confusion Assessment Method (CAM) [16] for delirium. Illness severity was defined using the systemic inflammatory response syndrome ( 2 or more of heart rate $>90$ beats per minute, temperature $<36$ or $>38^{\circ} \mathrm{C}$, respiratory rate $>20$ breaths per minute, white blood cell count $<4 \times 10^{9}$ or $>12 \times 10^{9}$ cells per litre) [17].

\section{Statistical Analysis}

Baseline characteristics of patients with any admission with delirium versus those without delirium were compared using $t$ test and ANOVA for continuous variables and $\chi^{2}$ for categorical variables. MMSE and MoCA cognitive test scores were obtained from the most recent OXVASC study follow-up performed prior to the hospital admission episode (Fig. 1). Cognitive test scores were dichotomized into mild (MMSE <27, MoCA <26) and moderate/ severe (MMSE $<24$, MoCA $<18$ ) cognitive impairment using previously described cut-offs [8].

To determine the associations between pre-admission OXVASC cognitive impairment (MMSE and MoCA scores) and delirium occurring during hospitalization, we used data from all admissions to calculate odds ratios (ORs). We adjusted for demographic factors and then for key covariates known to be associated with delirium in general cohorts that were available in our dataset $[1,2]$ : age, sex, and education (model 1), age, sex, education, history of depression at baseline OXVASC assessment, and illness severity on admission (model 2), and then with the addition of baseline OXVASC stroke severity (model 3). Similarly, we examined associations between individual MMSE and MoCA subtest scores and delirium adjusted for age, sex, and education. We also performed the following sensitivity analyses: 


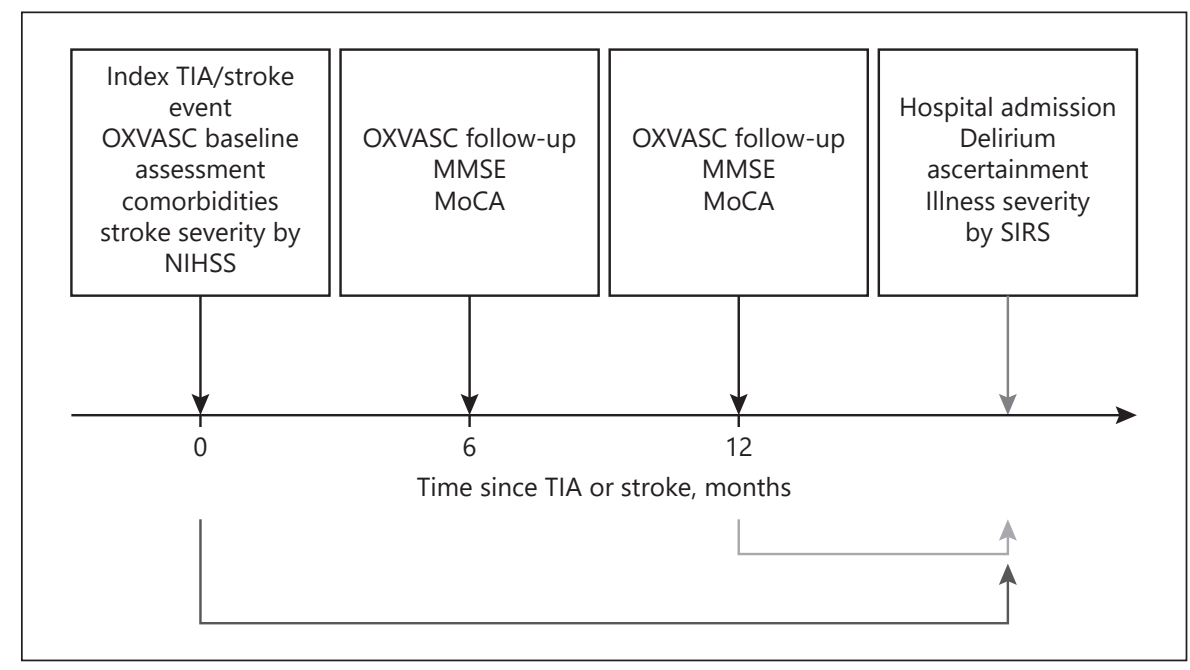

Fig. 1. Schematic diagram showing the design of the current study with the example of a patient who was hospitalized after their 12-month OXVASC follow-up and before their 5-year OXVASC follow-up. Time elapsed between OXVASC baseline TIA/stroke assessment to hospital admission is shown by the black curved arrow. NIHSS for stroke severity and comorbidities, including history of depression and vascular risk factors are acquired at baseline TIA/stroke assessment. Time between OXVASC follow-up MMSE

1. Substituting stroke severity in model 3 with the 1 month poststroke modified Rankin score or baseline stroke subtype as defined by the TOAST classification.

2. Adjusting model 3 further for admission characteristics defined as elective versus unplanned admission and treatment speciality.

3. Using data restricted to the first admission only.

\section{Results}

Among TIA and stroke patients recruited since April 2002 (mean age/SD = 68.9/13.3 years, 676 TIA) 1,565 were still alive on October 12, 2013. Over the subsequent study period, there was a total of 194 admissions (100 to OUHFT and 94 to EMU) in 158 OXVASC participants $(\mathrm{mean} / \mathrm{SD}$ age at admission $=79.5 / 11.2$ years, range $47-$ 100 years). The majority (170 [88\%]) of admissions were unplanned: $122(72 \%)$ to acute general (internal) medicine; 15 other medical services; 23 surgery; 10 trauma. Mean/SD time between baseline OXVASC TIA/stroke event and first hospital admission was 4.7/3.6 years and between most recent cognitive tests at OXVASC followup and first hospital admission was 1.7/1.8 years (Table 1).

Delirium occurred in 67/194 (34.5\%) admissions and $59 / 158(37 \%)$ patients had at least 1 admission compli- and MoCA testing and hospital admission is shown by the grey curved arrow. During hospital admission, delirium diagnosis was ascertained together with illness severity as defined by the SIRS criteria. OXVASC, Oxford Vascular Study; NIHSS, National Institutes of Health Stroke Scale; MMSE, mini-mental-state-examination; MoCA, Montreal Cognitive Assessment; SIRS, systemic inflammatory syndrome.

cated by delirium ( 53 had 1 episode, 4 had 2 episodes, and 2 had 3 episodes). Patients with versus without delirium were older $(\mathrm{mean} / \mathrm{SD}$ age $=84.9 / 8.9$ vs. $76.7 / 11.2$ years, $p<0.0001$ ) with more depression at OXVASC baseline $(21,31.3 \%$ vs. $19,15.0 \%, p=0.02$, adjusted for age $)$ and worse pre-admission cognition (mean/SD MMSE = 23.1/5.1 vs. 26.0/3.8, $p=0.002$ and mean/SD MoCA $18.8 / 6.1$ vs. $23.9 / 4.5, p<0.0001$, adjusted for age) but were similar in terms of sex, education, and vascular risk factors (Table 1).

Associations between pre-admission cognitive impairment and delirium were stronger for the MoCA $(\mathrm{OR}=1.2095 \%$ CI $1.11-1.30, p<0.0001$ per point decrease) than the MMSE (OR $=1.161 .07-1.27, p<0.0001)$ and for more severe versus less severe impairment (Table 2). Associations were robust to adjustment for demographic factors (model 1) with OR $=1.14,1.08-1.27$ ( $p=$ $0.005)$ for MMSE and OR $=1.16,1.06-1.25(p<0.0001)$ for MoCA and were largely unaffected by the addition of illness severity, depression history (model 2), and stroke severity (model 3, Table 2). Sensitivity analyses substituting post-stroke disability (modified Rankin score) or baseline stroke subtype for stroke severity in model 3 showed similar results as did further adjustment of model 3 for admission characteristics (elective vs. unplanned and treatment speciality, online suppl. Tables). Further 

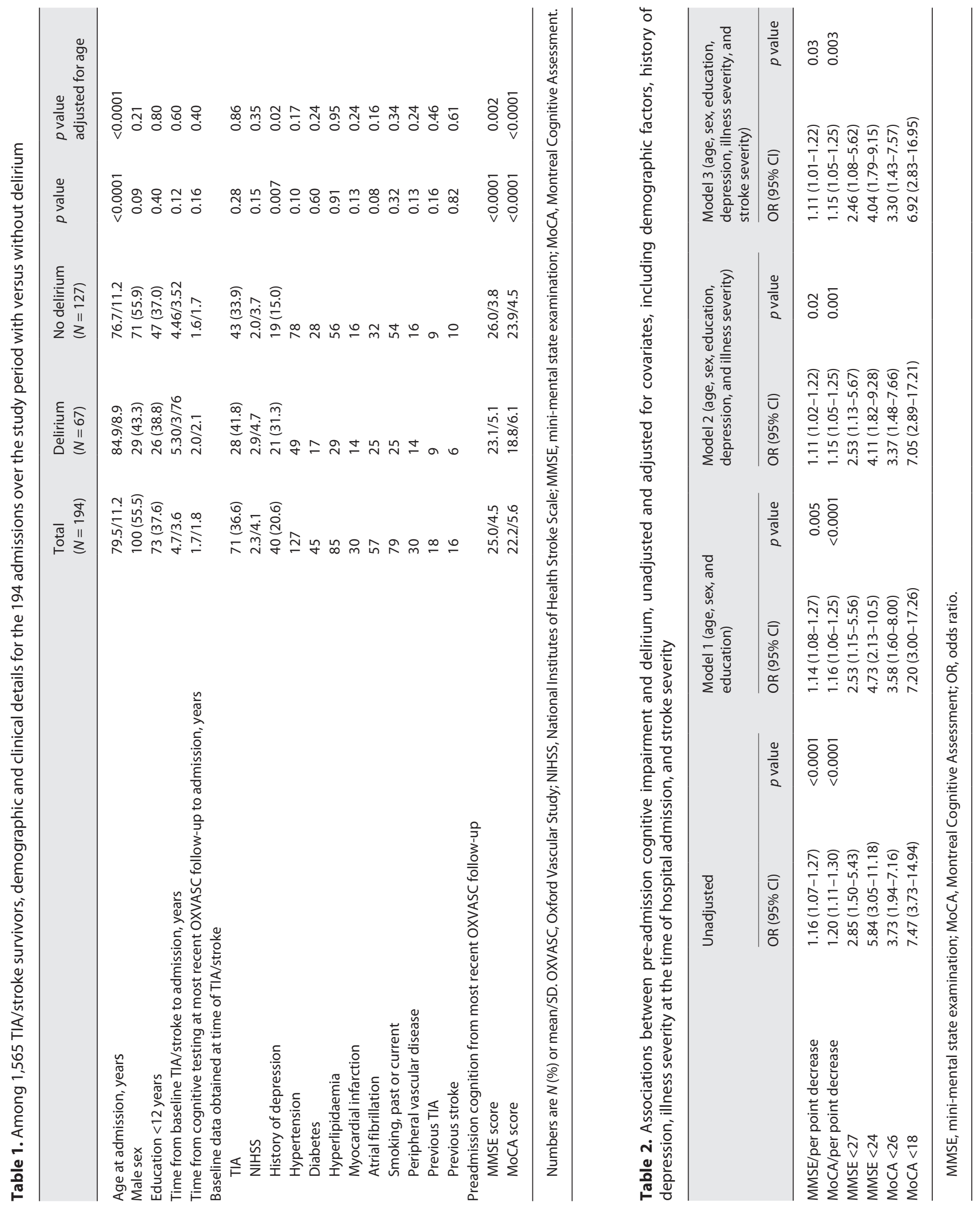
Table 3. MMSE and MoCA subtest scores measured at the time of most recent pre-admission OXVASC follow-up for all admissions with and without delirium adjusted for demographic factors (age, sex, and education)

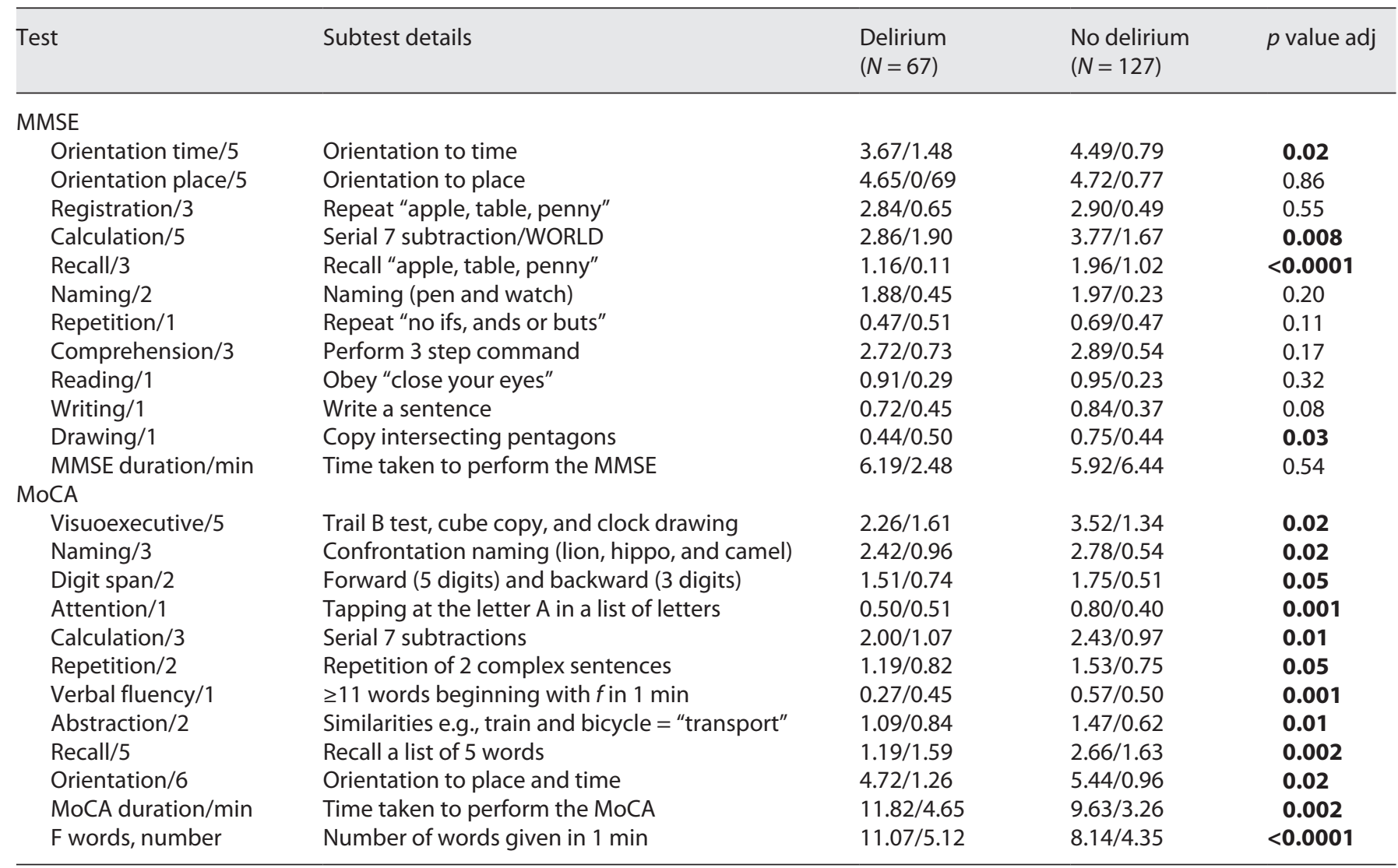

OXVASC, Oxford Vascular Study; MMSE, mini-mental-state-examination; MoCA, Montreal Cognitive Assessment. Bold values are significant.

sensitivity analyses using only the first admission only gave similar results (data not shown).

All 10 subtests on the MoCA and 4/11 on the MMSE were significantly associated with delirium (Table 3 ). Significant MMSE subtests were orientation to time $(p=$ $0.02)$, calculation $(p=0.008)$, recall $(p<0.0001)$, and copying pentagons ( $p=0.03$, Table 3 ; Fig. 2$)$. On the MoCA, the strongest associations were for sustained attention (tapping at the letter $\mathrm{A}, p=0.001$ ), verbal fluency ( $p=0.001$, total number of $\mathrm{f}$ words $p<0.0001)$, recall $(p=0.002)$, abstraction $(p=0.01)$, calculation $(p=0.01)$, visuo-executive function ( $p=0.02)$, naming $(p=0.02)$, and orientation ( $p=0.02$, Table 3 ; Fig. 2$)$. The time taken to perform the cognitive test was longer in those with versus without delirium for the MoCA (mean/SD $=11.8 / 4.7$ vs. $9.6 / 3.3 \mathrm{~min}, p=0.002)$ but not the MMSE $(6.2 / 2.5 \mathrm{vs}$. $5.9 / 6.4 \mathrm{~min}, p=0.54$ ). Sensitivity analyses using only the first admission gave similar results (data not shown).

\section{Discussion}

In our study of TIA/stroke patients on long-term follow-up, rates of delirium associated with hospitalization were high and were broadly similar to those reported in general hospital cohorts of older patients $[1,2]$. Pre-existing cognitive impairment of increasing severity, especially in frontal/executive and recall domains, predicted delirium in the long-term after TIA or stroke. Associations were robust to adjustment for other important factors including demographic characteristics, history of depression, and illness severity at the time of hospital admission and baseline stroke severity.

Studies in non-stroke cohorts have shown that delirium is more likely in patients with pre-existing cognitive impairment of increasing severity $[1,2]$. Since stroke-associated cognitive impairment is linked in a stepwise fashion to the severity of the stroke [3], we might have 


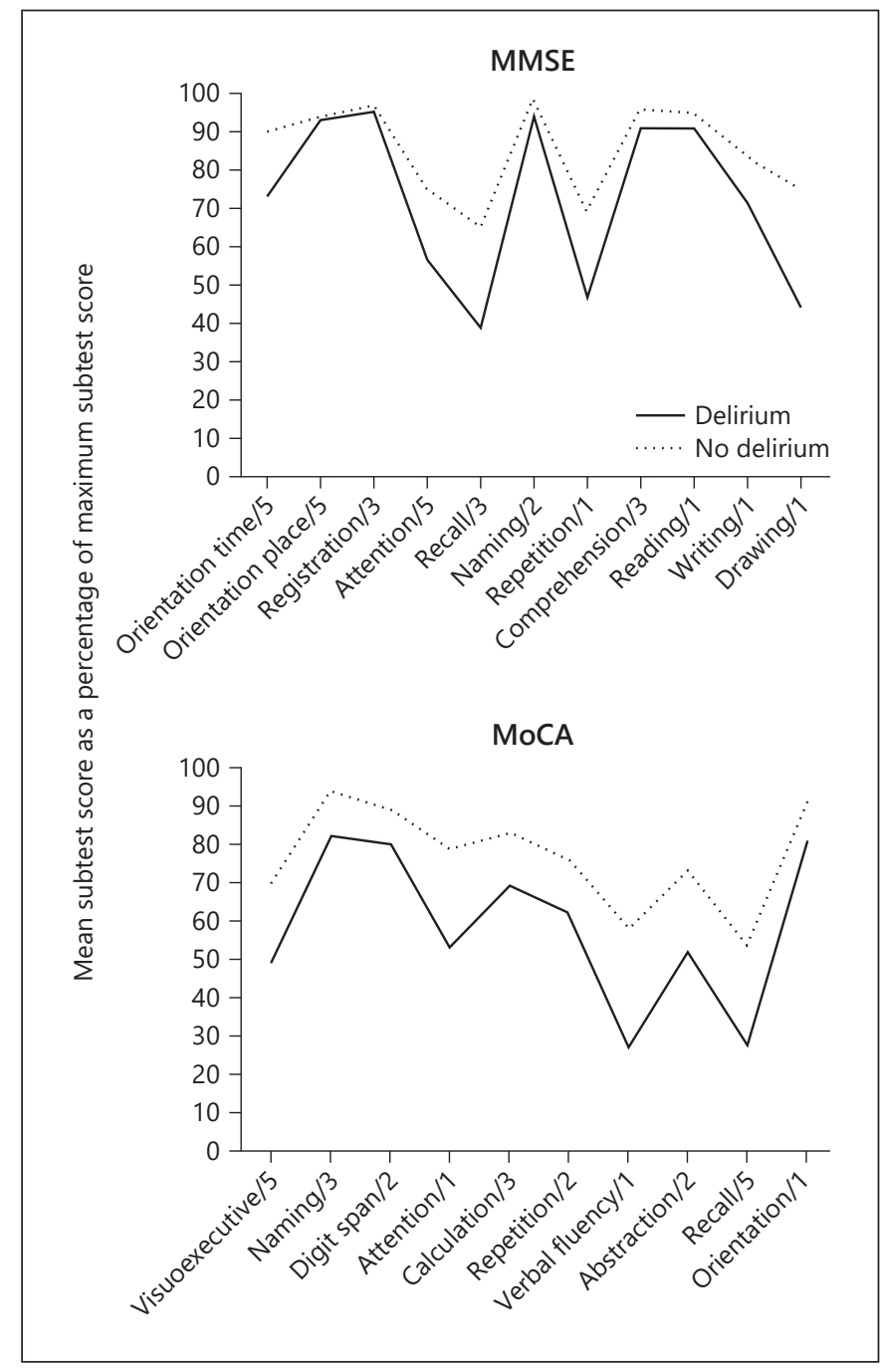

Fig. 2. Mean pre-admission MMSE (upper figure) and MoCA (lower figure) subtest scores shown as a percentage of the maximum subtest score, for patients with (dashed line) and without (solid line) delirium. MMSE, mini-mental-state-examination; MoCA, Montreal Cognitive Assessment.

expected less strong associations between preadmission cognition and delirium after adjustment for baseline stroke severity. In fact, this had little effect probably because the majority of survivors in our cohort had relatively minor strokes or TIA. Although the link between pre-existing cognitive impairment and delirium is wellestablished, there are few data on whether the underlying subtype of neuropathology is important. One small autopsy study failed to show a relationship between any specific neuropathological marker and delirium [18]. In contrast, patients with vascular versus Alzheimer's dementia appear more likely to get delirium [19] and are over-represented in hospital cohorts [13]. In addition, neuroimaging studies suggest a stronger role for white matter disease than neurodegeneration in mediating delirium risk [20].

In our study, we saw strong associations between deficits in frontal/executive domains and memory and delirium risk. The MoCA has broader coverage of frontal and attentional cognitive domains than the MMSE and more difficult memory and language items. Consequently, impairment in nearly all the MoCA subtests predicted future delirium compared to only 4 of the MMSE subtests. The typical "vascular pattern" cognitive impairment profile after stroke with frontal/executive and recall deficits contrasts with that of early Alzheimer's disease in which short-term recall is most affected [6, 7]. Previous data on cognitive domain impairment profile and delirium risk are largely from highly selected elective surgical populations. Almost all studies are concerned with shortterm delirium risk. Available data suggest associations with executive dysfunction: in 1 inclusive study of longterm (up to 1 year) prediction of delirium in older people living in residential care, strong associations with baseline Stroop test were found [21], although other studies have shown associations also with memory [22].

The mechanisms underlying the association between the cognitive domain impairment profile and delirium are unclear. Altered brain networks have been shown to underpin executive/attentional as well as memory deficits, and occur in stroke and neurodegenerative disease $[5,22]$. These network changes may predispose to network disintegration and the delirium syndrome in the face of precipitants including acute illness or environmental changes $[23,24]$. Alternatively, deficits in executive function and recall may associate with delirium because they are a clinical manifestation of the underlying cerebral pathology, including cerebrovascular disease burden, and associated blood-brain barrier permeability. Damage to the blood-brain barrier is thought to be an important mechanism in delirium through enabling inflammatory mediators to reach the brain [25].

Strengths of our study include the nesting within an on-going inclusive longitudinal population-based cohort study enriched for cerebrovascular disease with prospective patient evaluation for delirium. The study design allowed prospective assessment of pre-admission cognitive function using validated tests and robust adjustment for confounders often not considered in previous studies. Limitations include the use of cognitive screening tests rather than a comprehensive neuropsychological battery, although this enabled inclusion of older more impaired 
patients at greater risk of delirium. In-depth neuropsychological assessment may have provided more detail on the pattern of cognitive deficits contributing to delirium vulnerability. In addition, we did not ascertain episodes of delirium occurring in non-hospitalized patients in the community. There are few existing data but community prevalence of $<0.5 \%$ has been reported in older people without dementia [26]. Since delirium is a powerful driver of hospital admission even in those in long-term care $[1,26,27]$, it is unlikely that there were large numbers of delirium cases in the community without admission. Also, we did not examine the precipitating factors for delirium in detail but the breakdown by admission type suggested that acute medical emergencies were important. Finally, we were only able to prospectively collect data on hospital admissions over a 6 month snapshot period rather than over the entire OXVASC study timeframe which would have enabled us to undertake a linear mixed-effects analysis increasing power and fully exploiting the longitudinal nature of OXVASC. The move to electronic patient records and better routine documentation of delirium may make this possible in future studies.

\section{Conclusion}

TIA/stroke-associated cognitive impairment increases the risk of future delirium with particular importance of deficits in frontal/executive function and recall. Further work is needed to establish the underlying mechanisms and whether inclusion of cognitive impairment profile improves the accuracy of delirium risk models [28]. Future large studies are required to enable mixedeffects modelling to determine the impact of delirium occurrence on future dementia risk. Long-term delirium risk and acute delirium at the time of stroke should be considered as part of the overall burden of TIA and stroke.

\section{Acknowledgment}

We acknowledge the use of the Acute Vascular Imaging Centre, Oxford.

\section{Statement of Ethics}

This research was conducted ethically in accordance with the World Medical Association Declaration of Helsinki. The Oxford Vascular Study is approved by the local research Ethics Committee
(OxREC No. C02.043). Informed written consent (or assent from relatives) was obtained for study interview and face-to-face and telephone follow-up as well as indirect follow-up using medical records.

\section{Conflict of Interest Statement}

S.T.P. has received royalties from Oxford University Press and Cambridge University Press and Consultancy Fees from University of Michigan, USA and honoraria from the University of Trondheim Norway, La Trobe University, Melbourne, Australia, and the University of Sydney, Australia. R.J.T. and S.J.V.W. have nothing to declare. P.M.R. has received royalties from Oxford University Press and Cambridge University Press and honoraria for trial committee work from Bayer, Leverkusen, Germany and BMS.

\section{Funding Sources}

The Oxford Vascular Study has been funded by the Wellcome Trust, Wolfson Foundation, UK Stroke Association, British Heart Foundation, Dunhill Medical Trust, National Institute of Health Research (NIHR), Medical Research Council, and the NIHR Oxford Biomedical Research Centre. S.T.P. is supported by the NIHR Oxford Biomedical Research Centre. P.M.R. is an emeritus NIHR Senior investigator and a Wellcome Trust Investigator.

\section{Author Contributions}

S.T.P. designed this substudy, cleaned and assembled data, performed analyses, and wrote the manuscript. S.T.P. also developed, validated, and implemented the Oxford University Hospitals NHS Foundation Trust cognitive screen, as part of her role as Clinical Lead for Dementia and Delirium. R.J.T. and S.J.V.W. collected data. P.M.R. had set up and directed the Oxford Vascular Study and provided critical input to the analyses and manuscript.

\section{Data Availability Statement}

Applications for access to OXVASC study data will be considered by P.M.R. (peter.rothwell@ndcn.ox.ac.uk).

\section{Disclaimer}

The views expressed are those of the authors and not necessarily those of the NHS, the NIHR, or the Department of Health and Social Care. 


\section{References}

1 Wilson JE, Mart MF, Cunningham C, Shehabi Y, Girard TD, MacLullich AMJ, et al. Delirium. Nat Rev Dis Primers. 2020;6(1):90.

2 Pendlebury ST, Lovett NG, Smith SC, Dutta N, Bendon C, Lloyd-Lavery A, et al. Observational, longitudinal study of delirium in consecutive unselected acute medical admissions: age-specific rates and associated factors, mortality and re-admission. BMJ Open. 2015;5: e007808.

3 Pendlebury ST, Rothwell PM. Incidence and prevalence of dementia associated with transient ischaemic attack and stroke: analysis of the population-based Oxford Vascular Study. Lancet Neurol. 2019;18:248-58.

4 Rudolph JL, Jones RN, Grande LJ, Milberg WP, King EG, Lipsitz LA, et al. Impaired executive function is associated with delirium after coronary artery bypass graft surgery. J Am Geriatr Soc. 2006;54:937-41.

5 Gold BT, Brown CA, Hakun JG, Shaw LM, Trojanowski JQ, Smith CD. Clinically silent Alzheimer's and vascular pathologies influence brain networks supporting executive function in healthy older adults. Neurobiol Aging. 2017;58:102-11.

6 Pendlebury ST, Markwick A, de Jager CA, Zamboni G, Wilcock GK, Rothwell PM. Differences in cognitive profile between TIA, stroke and elderly memory research subjects: a comparison of the MMSE and MoCA. Cerebrovasc Dis. 2012;34:48-54.

7 Sachdev PS, Brodaty H, Valenzuela MJ, Lorentz L, Looi JC, Wen W, et al. The neuropsychological profile of vascular cognitive impairment in stroke and TIA patients. Neurology. 2004;62:912-9.

8 Pendlebury ST, Mariz J, Bull L, Mehta Z, Rothwell PM. MoCA, ACE-R, and MMSE versus the National Institute of Neurological Disorders and Stroke-Canadian Stroke Network Vascular Cognitive Impairment Harmonization Standards Neuropsychological Battery after TIA and stroke. Stroke. 2012;43: 464-9.

9 Oldenbeuving AW, de Kort PL, Jansen BP, Algra A, Kappelle LJ, Roks G. Delirium in the acute phase after stroke: incidence, risk factors, and outcome. Neurology. 2011;76:9939.
10 Rothwell PM, Coull AJ, Giles MF, Howard SC, Silver LE, Bull LM, et al. Change in stroke incidence, mortality, case-fatality, severity, and risk factors in Oxfordshire, UK from 1981 to 2004 (Oxford Vascular Study). Lancet. 2004;363:1925-33.

11 Folstein MF, Folstein SE, McHugh PR. "Minimental state". A practical method for grading the cognitive state of patients for the clinician. J Psychiatr Res. 1975;12:189-98.

12 Nasreddine ZS, Phillips NA, Bédirian V, Charbonneau S, Whitehead V, Collin I, et al. The Montreal Cognitive Assessment, MoCA: a brief screening tool for mild cognitive impairment. J Am Geriatr Soc. 2005;53:695-9.

13 Pendlebury ST, Lovett NG, Thomson RJ, Smith SC. Impact of a system-wide multicomponent intervention on administrative diagnostic coding for delirium and other cognitive frailty syndromes: observational prospective study. Clin Med. 2020;20:454-64.

14 American Psychiatric Association. Diagnostic and statistical manual of mental disorders (DSM-IV). 4th ed. Washington: American Psychiatric Association; 1994.

15 Hodkinson HM. Evaluation of a mental test score for assessment of mental impairment in the elderly. Age Ageing. 1972;1:233-8.

16 Inouye SK, van Dyck CH, Alessi CA, Balkin S, Siegal AP, Horwitz RI. Clarifying confusion: the confusion assessment method. A new method for detection of delirium. Ann Intern Med. 1990;113:941-8.

17 Bone RC, Balk RA, Cerra FB, Dellinger RP, Fein AM, Knaus WA, et al. American College of Chest Physicians/Society of Critical Care Medicine Consensus Conference: definitions for sepsis and organ failure and guidelines for the use of innovative therapies in sepsis. Crit Care Med 1992;20:864-74.

18 Davis DH, Muniz Terrera G, Keage H, Rahkonen T, Oinas M, Matthews FE, et al. Delirium is a strong risk factor for dementia in the oldest-old: a population-based cohort study. Brain. 2012;135:2809-16.

19 Hasegawa N, Hashimoto M, Yuuki S, Honda $\mathrm{K}$, Yatabe Y, Araki K, et al. Prevalence of delirium among outpatients with dementia. Int Psychogeriatr. 2013;25:1877-83.
20 Nitchingham A, Kumar V, Shenkin S, Ferguson KJ, Caplan GA. A systematic review of neuroimaging in delirium: predictors, correlates and consequences. Int J Geriatr Psychiatry. 2018;33:1458-78.

21 Katz IR, Curyto KJ, TenHave T, Mossey J, Sands L, Kallan MJ. Validating the diagnosis of delirium and evaluating its association with deterioration over a one-year period. Am J Geriatr Psychiatry. 2001;9:148-59.

22 Jankowski CJ, Trenerry MR, Cook DJ, Buenvenida SL, Stevens SR, Schroeder DR, et al. Cognitive and functional predictors and sequelae of postoperative delirium in elderly patients undergoing elective joint arthroplasty. Anesth Analg. 2011;112:1186-93.

23 Cheng B, Schlemm E, Schulz R, Boenstrup M, Messé A, Hilgetag C, et al. Altered topology of large-scale structural brain networks in chronic stroke. Brain Commun. 2019; 1: fcz020.

24 van Montfort SJT, van Dellen E, Stam CJ, et al. Brain network disintegration as a final common pathway for delirium: a systematic review and qualitative meta-analysis. Neuroimage Clin. 2019;23:101809.

25 van Gool WA, van de Beek D, Eikelenboom P. Systemic infection and delirium: when cytokines and acetylcholine collide. Lancet. 2010;375:773-5.

26 Andrew MK, Freter SH, Rockwood K. Prevalence and outcomes of delirium in community and non-acute care settings in people without dementia: a report from the Canadian Study of Health and Aging. BMC Med. 2006;4:15.

27 Elias TCN, Bowen J, Hassanzadeh R, Lasserson DS, Pendlebury ST. Factors associated with admission to bed-based care: observational prospective cohort study in a multidisciplinary same day emergency care unit (SDEC). BMC Geriatr. 2021;21(1):8.

28 Pendlebury ST, Lovett NG, Smith SC, Wharton R, Rothwell PM. Delirium risk stratification in consecutive unselected admissions to acute medicine: validation of a susceptibility score based on factors identified externally in pooled data for use at entry to the acute care pathway. Age Ageing. 2017;46:226-31. 